\title{
Solution of Linear Dynamical Systems Using Lucas Polynomials of the Second Kind
}

\author{
Pierpaolo Natalini'1, Paolo E. Ricci² \\ ${ }^{1}$ Dipartimento di Matematica e Fisica, Largo San Leonardo Murialdo, Università degli Studi Roma Tre, Roma, \\ Italia \\ ${ }^{2}$ International Telematic University UniNettuno, Roma, Italia \\ Email:natalini@mat.uniroma3.it,paoloemilioricci@gmail.com
}

Received 23 February 2016; accepted 24 April 2016; published 27 April 2016

Copyright (C) 2016 by authors and Scientific Research Publishing Inc.

This work is licensed under the Creative Commons Attribution International License (CC BY).

http://creativecommons.org/licenses/by/4.0/

(c) (i) Open Access

\section{Abstract}

The use of $F_{k, n}$ functions, expressible in terms of Lucas polynomials of the second kind, allows us to write down the solution of linear dynamical systems-both in the discrete and continuous case-avoiding the Jordan canonical form of involved matrices. This improves the computational complexity of the algorithms used in literature.

\section{Keywords}

\author{
Matrix Powers, Linear Dynamical Systems, Exponential Matrix, Lucas Polynomials of the Second \\ Kind
}

\section{Introduction}

Even in recent books (see e.g. [1] [2]), the solution of linear dynamical systems, both in the discrete or continuous time case, is expressed by using all powers of the considered matrix $\mathcal{A}_{r \times r}$. As a consequence, if we want to write down explicitly the solution, it is necessary to construct the Jordan canonical form of $\mathcal{A}$ and, in the case of a defective matrix (i.e. if non trivial Jordan blocks appear in its canonical form), this implies cumbersome computations.

In order to avoid this serious problem, we propose here an alternative method, based on recursion, using the $F_{k, n}$ functions, which are essentially linked to Lucas polynomials of the second kind [3] (i.e. the basic solution of a homogeneous linear recurrence relation with constant coefficients [4] [5]), and to the multi-variable Chebyshev polynomials [6].

After recalling the $F_{k, n}$ functions and their connections with matrix powers [7], we can show, in Section 2, that the use of matrix powers and matrix function representations (see e.g. [7] [8]) gives us the possibility to use 
only powers of the considered matrix up to (at most) the order $r-1$. This is a trivial consequence of the Cayley-Hamilton theorem, and should be used, in our opinion, to reduce the computational cost of solutions. Another shown possibility is the use of the Riesz-Fantappiè formula, by means of which the Taylor expansion of solution is completely avoided.

In Section 3, we prove our main results, relevant to an alternative method for the solution of linear dynamical systems, both in the discrete and continuous time case and via the Riesz-Fantappiè formula, also known in literature as the Dunford-Schwartz formula [9], (but the priority of the first Authors is undubtable).

Some concrete examples of computation are presented in Section 4, showing the more simple complexity of our procedure with respect to the traditional algorithms, as they appear in the above mentioned books.

We want to remark explicitly that, in our article, by using the $F_{k, n}$ functions (essentially linked to Lucas polynomials of the second kind), our methodology builds a bridge, to our knowledge not previously well known, between the Theory of Matrices and that of Special Functions, which are usually considered as very different fields. Furthermore, the use of the Riesz-Fantappiè formula reduces to a finite computation the algorithms used in literature, making use of series expansions, and consequently dramatically improves the computation complexity of the considered problem.

\subsection{Recalling $F_{k, n}$ Functions}

Consider the $(r+1)$-terms homogeneous linear bilateral recurrence relation with (real or complex) constant (with respect to $n$ ) coefficients $u_{k}(k=1,2, \cdots, r)$ where $u_{r} \neq 0$ :

$$
X_{n}=u_{1} X_{n-1}-u_{2} X_{n-2}+\cdots+(-1)^{r-1} u_{r} X_{n-r}, \quad(n \in \mathbf{Z}) .
$$

Supposing the coefficients vary, its solution is given by every bilateral sequence $\left\{X_{n}\left(u_{1}, u_{2}, \cdots, u_{r}\right)\right\}_{n \in \mathbf{Z}}$ such that $r+1$ consecutive terms satisfy Equation (1.1).

A basis for the $r$-dimensional vectorial space $\mathcal{V}_{r}$ of solutions is given by the functions

$F_{k, n}=F_{k, n}\left(u_{1}, u_{2}, \cdots, u_{r}\right), \quad(k=1,2, \cdots, r, n \geq-1)$, defined by the initial conditions below:

$$
\begin{array}{cccc}
F_{1,-1}=0 & F_{1,0}=0 & \ldots & F_{1, r-2}=1, \\
F_{2,-1}=0 & F_{2,0}=1 & \ldots & F_{2, r-2}=0, \\
\vdots & \vdots & & \vdots \\
F_{r,-1}=1 & F_{r, 0}=0 & \ldots & F_{r, r-2}=0 .
\end{array}
$$

Since $u_{r} \neq 0$, the $F_{k, n}$ functions can be defined even if $n<-1$, by means of the positions:

$$
F_{k, n}\left(u_{1}, \cdots, u_{r}\right)=F_{r-k+1,-n+r-3}\left(\frac{u_{r-1}}{u_{r}}, \cdots, \frac{u_{1}}{u_{r}}, \frac{1}{u_{r}}\right), \quad(k=1, \cdots, r ; n \in \mathbf{Z}) .
$$

Therefore, assuming the initial conditions $X_{j}, X_{j+1}, \cdots, X_{j+r-1}$, the general solution of the recurrence (1.1), $\forall n \in \mathbf{Z}$, is given by

$$
X_{n+j+1}=X_{j} F_{r, n}+X_{j+1} F_{r-1, n}+\cdots+X_{j+r-1} F_{1, n} . \quad(n \in \mathbf{Z}) .
$$

For further considerations, relevant to the classical method for solving the recurrence (1.1), see [5].

An important result, originally stated by É Lucas [3] (in the particular case $r=2$ ), is given by the equations

$$
\left\{\begin{array}{l}
F_{1, n}=u_{1} F_{1, n-1}+F_{2, n-1} \\
F_{2, n}=-u_{2} F_{1, n-1}+F_{3, n-1} \\
\quad \vdots \\
F_{r-1, n}=(-1)^{r-2} u_{r-1} F_{1, n-1}+F_{r, n-1} \\
F_{r, n}=(-1)^{r-1} u_{r} F_{1, n-1}
\end{array}\right.
$$

showing that all $\left\{F_{k, n}\right\}_{n \in \mathbf{Z}}$ functions are expressed through the only bilateral sequence $\left\{F_{1, n}\right\}_{n \in \mathbf{Z}}$.

Therefore, we assume the following 
Definition 1-The bilateral sequence $\left\{F_{1, n}\right\}_{n \in \mathbf{Z}}$, solution of (1.1) corresponding to the initial conditions:

$$
F_{1,-1}=0, F_{1,0}=0, \cdots, F_{1, r-3}=0, F_{1, r-2}=1,
$$

is called the fundamental solution of (1.1) (“fonction fondamentale” by É. Lucas [3]), [4].

For the connection with Chebyshev polynomials of the second kind in several variables, see [6].

\subsection{Matrix Powers Representation}

In preceding articles [5] [7], the following result is proved:

Theorem 1 Given an $r \times r$ matrix $\mathcal{A}$, putting by definition $u_{0}:=1$, and denoting by

$$
P(\lambda):=\operatorname{det}(\lambda \mathcal{I}-\mathcal{A})=\sum_{j=0}^{r}(-1)^{j} u_{j} \lambda^{r-j}
$$

its characteristic polynomial (or possibly its minimal polynomial, if this is known), the matrix powers $\mathcal{A}^{n}$, with integral exponent $n$, are given by the equation:

$$
\mathcal{A}^{n}=F_{1, n-1}\left(u_{1}, \cdots, u_{r}\right) A^{r-1}+F_{2, n-1}\left(u_{1}, \cdots, u_{r}\right) \mathcal{A}^{r-2}+\cdots+F_{r, n-1}\left(u_{1}, \cdots, u_{r}\right) \mathcal{I},
$$

where the functions $F_{k, n}\left(u_{1}, \cdots, u_{r}\right)$ are defined in Section 1.1.

Moreover, if $\mathcal{A}$ is not singular, i.e. $u_{r} \neq 0$, Equation (1.3) still works for negative integers $n$, assuming the definition (1.2) for the $F_{k, n}$ functions.

It is worth to recall that the knowledge of eigenvalues is equivalent to that of invariants, since the second ones are the elementary symmetric functions of the first ones.

Remark 1 Note that, as a consequence of the above result, the higher powers of matrix $\mathcal{A}$ are always expressible in terms of the lower ones (at most up to the dimension of $\mathcal{A}$ ).

\section{Matrix Functions Representation}

It is well known that an analytic function $f$ of a matrix $\mathcal{A}=\mathcal{A}_{r \times r}$, i.e. $f(\mathcal{A})$, is the matrix polynomial obtained from the scalar polynomial interpolating the function $f$ on the eigenvalues of $\mathcal{A}$ (see e.g. the Gantmacher book [8]), however, in many books (see e.g. [1]), the series expansion

$$
\exp \mathcal{A}=\sum_{n=0}^{\infty} \frac{\mathcal{A}^{n}}{n !}
$$

is assumed for defining (and computing) $\exp (\mathcal{A})$. So, apparently, the series expansion for the exponential of a matrix is "hard to die".

Let $S_{\mathcal{A}}=\left\{\lambda_{1}, \lambda_{2}, \cdots, \lambda_{r}\right\}$ the spectrum of $\mathcal{A}$. Denoting by

$$
\Pi_{r-1}(x)=\alpha_{0}+\alpha_{1} x+\cdots+\alpha_{r-1} x^{r-1}
$$

the polynomial interpolating $\exp (x)$ on $S_{\mathcal{A}}$, i.e. such that: $\Pi_{r-1}\left(\lambda_{i}\right)=\exp \left(\lambda_{i}\right)$,

$(i=1,2, \cdots, r)$, then

$$
\exp \mathcal{A}=\alpha_{0} \mathcal{I}+\alpha_{1} \mathcal{A}+\cdots+\alpha_{r-1} \mathcal{A}^{r-1} .
$$

If the eigenvalues are all distinct, $\Pi_{r-1}$ coincides with the Lagrange interpolation polynomial and (2.2) is the Lagrange-Sylvester formula. In case of multiple eigenvalues, $\Pi_{r-1}$ is the Hermite interpolation polynomial, and (2.2) reduces to Arthur Buchheim's formula, generalizing the preceding one.

This avoids the use of higher powers of $\mathcal{A}$ in the Taylor expansion (2.1). In any case, the possibility to write $\mathcal{A}^{h},(h \geq 2)$, in an easy block form, requires not only the knowledge of the spectrum, but even the Jordan canonical form of $\mathcal{A}$. It is necessary to compute the eigenvectors and moreover the principal vectors, if $\mathcal{A}$ is defective. A known machinery which implies a lot of computations.

\section{The Riesz-Fantappiè Formula}

A classical result is as follows: 
Theorem 2 Under the hypotheses and definitions considered above, the resolvent matrix $(\lambda \mathcal{I}-\mathcal{A})^{-1}$ can be represented as

$$
(\lambda \mathcal{I}-\mathcal{A})^{-1}=\frac{1}{P(\lambda)} \sum_{h=0}^{r-1}\left[\sum_{j=0}^{r-h-1}(-1)^{j} u_{j} \lambda^{r-h-j-1}\right] \mathcal{A}^{h} .
$$

Then, by the Riesz-Fantappiè formula, we recover the classical result:

Theorem 3 If $f(\lambda)$ is a holomorphic function in the domain $\mathcal{C}$ and denoting by $D \subset \mathcal{C}$ (with $\bar{D} \subset \mathcal{C}$ ) a closed set whose boundary is a piecewise simple Jordan contour $\gamma$ encompassing the spectrum $S_{\mathcal{A}}$ of $\mathcal{A}$, the matrix function $f(\mathcal{A})$ can be represented by:

$$
\begin{aligned}
f(\mathcal{A}) & =\frac{1}{2 \pi i} \oint_{\gamma} f(\lambda)(\lambda \mathcal{I}-\mathcal{A})^{-1} \mathrm{~d} \lambda \\
& =\sum_{h=0}^{r-1}\left[\frac{1}{2 \pi i} \sum_{j=0}^{r-h-1}(-1)^{j} u_{j} \oint_{\gamma} \frac{f(\lambda) \lambda^{r-h-j-1}}{P(\lambda)} \mathrm{d} \lambda\right] \mathcal{A}^{h} .
\end{aligned}
$$

In particular:

$$
\mathrm{e}^{\mathcal{A}}=\sum_{h=0}^{r-1}\left[\frac{1}{2 \pi i} \sum_{j=0}^{r-h-1}(-1)^{j} u_{j} \oint_{\gamma} \frac{\mathrm{e}^{\lambda} \lambda^{r-h-j-1}}{P(\lambda)} \mathrm{d} \lambda\right] \mathcal{A}^{h} .
$$

Remark 2 If the eigenvalues of $\mathcal{A}$, are known, Equation (2.3), by the residue theorem, gives back the Lagrange-Sylvester representation. However, for computing the integrals appearing in Equation (2.3) it is sufficient the knowledge of a circle $D,(\gamma:=\partial D)$, containing the spectrum of $\mathcal{A}$ (by using the Gerschgorin theorem, and then knowing only the entries of $\mathcal{A}$, without computing its eigenvalues). Therefore, this approach is computationally more convenient with respect to the Lagrange-Sylvester formula.

\section{Solution of Linear Dynamical Systems Via $F_{k, n}$ Functions}

As a consequence of the above recalled results, we can prove our main results both in the discrete and continuous time case.

\subsection{The Discrete Time Case}

Theorem 4 Consider the dynamical problem for the homogeneous linear recurrence system

$$
\left\{\begin{array}{l}
Z_{i+1}=\mathcal{A} \cdot Z_{i} \\
Z_{i=0}=Z_{0}
\end{array}\right.
$$

where

$$
\mathcal{A}=\mathcal{A}_{r \times r}=\left(\begin{array}{ccc}
a_{1,1} & \cdots & a_{1, r} \\
\vdots & \ddots & \vdots \\
a_{r, 1} & \cdots & a_{r, r}
\end{array}\right)
$$

Let

$$
\begin{gathered}
Z_{n}=\left(z_{1}^{(n)}, z_{2}^{(n)}, \cdots, z_{r}^{(n)}\right)^{\mathrm{T}}, \\
Z_{0}=\left(z_{1}^{(0)}, z_{2}^{(0)}, \cdots, z_{r}^{(0)}\right)^{\mathrm{T}}, \\
Z_{1}=\left(z_{1}^{(1)}, z_{2}^{(1)}, \cdots, z_{r}^{(1)}\right)^{\mathrm{T}}=\mathcal{A} \cdot Z_{0}, \\
\cdots \\
Z_{r-1}=\left(z_{1}^{(r-1)}, z_{2}^{(r-1)}, \cdots, z_{r}^{(r-1)}\right)^{\mathrm{T}}=\mathcal{A} \cdot Z_{0}^{r-2},
\end{gathered}
$$


denote by $u_{1}:=\operatorname{tr}(\mathcal{A}), u_{2}, \cdots, u_{r}:=\operatorname{det}(\mathcal{A})$ the invariants of $\mathcal{A}$, and recall the generalized Lucas polynomials $F_{k, n}\left(u_{1}, \cdots, u_{r}\right), \quad(k=1,2, \cdots, r, n \geq-1)$, defined in Section 1.1.

Define the vector

$$
\mathcal{F}_{n-1}\left(u_{1}, \cdots, u_{r}\right):=\left(F_{1, n-1}\left(u_{1}, \cdots, u_{r}\right), F_{2, n-1}\left(u_{1}, \cdots, u_{r}\right), \cdots, F_{r, n-1}\left(u_{1}, \cdots, u_{r}\right)\right)^{\mathrm{T}},
$$

and the matrix

$$
\left(Z_{r-1}, Z_{r-2}, \cdots, Z_{0}\right)=\left(\begin{array}{cccc}
z_{1}^{(r-1)} & z_{1}^{(r-2)} & \cdots & z_{1}^{(0)} \\
z_{2}^{(r-1)} & z_{2}^{(r-2)} & \cdots & z_{2}^{(0)} \\
\vdots & \vdots & \ddots & \vdots \\
z_{r}^{(r-1)} & z_{r}^{(r-2)} & \cdots & z_{r}^{(0)}
\end{array}\right)
$$

then, the solution of problem (3.1) can be written

$$
Z_{n}=\left(Z_{r-1}, Z_{r-2}, \cdots, Z_{0}\right) \cdot \mathcal{F}_{n-1}\left(u_{1}, \cdots, u_{r}\right) .
$$

That is, for the components:

$$
\begin{aligned}
z_{k}^{(n)} & =F_{1, n-1}\left(u_{1}, \cdots, u_{r}\right) z_{k}^{(r-1)}+F_{2, n-1}\left(u_{1}, \cdots, u_{r}\right) z_{k}^{(r-2)}+\cdots+F_{r, n-1}\left(u_{1}, \cdots, u_{r}\right) z_{k}^{(0)} \\
& =\sum_{h=1}^{r} F_{h, n-1}\left(u_{1}, \cdots, u_{r}\right) z_{k}^{(r-h)} .
\end{aligned}
$$

Proof It is well known that the solution of problem (3.1) is given by

$$
Z(t)=\mathcal{A}^{n} Z_{0} .
$$

From the results about matrix powers, it follows that

$$
Z(t)=\left(F_{1, n-1}\left(u_{1}, \cdots, u_{r}\right) \mathcal{A}^{r-1}+F_{2, n-1}\left(u_{1}, \cdots, u_{r}\right) \mathcal{A}^{r-2}+\cdots+F_{r, n-1}\left(u_{1}, \cdots, u_{r}\right) \mathcal{I}\right) Z_{0} .
$$

Then, taking into account the above definitions of vectors $\mathcal{F}_{n-1}\left(u_{1}, \cdots, u_{r}\right)$ and $Z_{1}, Z_{2}, \cdots, Z_{r-1}$ our result follows.

Remark 3 Note that, even if this is unrealistic, solution (3.2) still holds for negative values of $n$, assuming definition (1.2) for the $F_{k, n}$ functions when $n<-1$.

\subsection{The Continuous Time Case}

Theorem 5 Consider the Cauchy problem for the homogeneous linear differential system

$$
\left\{\begin{array}{l}
Z^{\prime}(t)=\mathcal{A} \cdot Z(t) \\
Z(0)=Z_{0},
\end{array}\right.
$$

where $\mathcal{A}=\mathcal{A}_{r \times r}$ is the same matrix considered in the discrete time case.

Let

$$
\begin{gathered}
Z(t)=\left(Z_{1}(t), Z_{2}(t), \cdots, Z_{r}(t)\right)^{\mathrm{T}}, \\
Z_{0}=Z_{0}^{0}=\left(Z_{1}(0), Z_{2}(0), \cdots, Z_{r}(0)\right)^{\mathrm{T}}, \\
Z_{0}^{\prime}=\left(Z_{1}^{\prime}(0), Z_{2}^{\prime}(0), \cdots, Z_{r}^{\prime}(0)\right)^{\mathrm{T}}=\mathcal{A} \cdot Z_{0}, \\
\cdots \\
Z_{0}^{r-1}=\left(Z_{1}^{r-1}(0), Z_{2}^{r-1}(0), \cdots, Z_{r}^{r-1}(0)\right)^{\mathrm{T}}=\mathcal{A} \cdot Z_{0}^{r-2},
\end{gathered}
$$

denote by $u_{1}:=\operatorname{tr}(\mathcal{A}), u_{2}, \cdots, u_{r}:=\operatorname{det}(\mathcal{A})$ the invariants of $\mathcal{A}$, and recall again the generalized Lucas polynomials $F_{k, n}\left(u_{1}, \cdots, u_{r}\right),(k=1,2, \cdots, r, n \geq-1)$. 
Introduce the matrix $\left(Z_{0}^{r-1}, \cdots, Z_{0}^{\prime}, Z_{0}\right)$ and define the vector function

$$
\mathcal{F}_{n-1}\left(u_{1}, \cdots, u_{r}\right):=\left(F_{1, n-1}\left(u_{1}, \cdots, u_{r}\right), F_{2, n-1}\left(u_{1}, \cdots, u_{r}\right), \cdots, F_{r, n-1}\left(u_{1}, \cdots, u_{r}\right)\right)^{\mathrm{T}} \text {, }
$$

then, the solution of problem (3.3) can be written

$$
Z(t)=\sum_{n=0}^{\infty}\left(Z_{0}^{r-1}, \cdots, Z_{0}^{\prime}, Z_{0}\right) \cdot \mathcal{F}_{n-1}\left(u_{1}, \cdots, u_{r}\right) \frac{t^{n}}{n !} .
$$

Proof-It is well known that the solution of problem (3.3) is given by

$$
Z(t)=\mathrm{e}^{t \mathcal{A}} Z_{0} \text {. }
$$

From the results about matrix exponential, it follows that

$$
\mathrm{e}^{t \mathcal{A}}=\sum_{n=0}^{\infty} \frac{(t \mathcal{A})^{n}}{n !}=\sum_{n=0}^{\infty} \mathcal{A}^{n} \frac{t^{n}}{n !},
$$

where

$$
\mathcal{A}^{n}=F_{1, n-1}\left(u_{1}, \cdots, u_{r}\right) \mathcal{A}^{r-1}+F_{2, n-1}\left(u_{1}, \cdots, u_{r}\right) \mathcal{A}^{r-2}+\cdots+F_{r, n-1}\left(u_{1}, \cdots, u_{r}\right) \mathcal{I},
$$

so that Equation (3.5) becomes

$$
Z(t)=\sum_{n=0}^{\infty}\left(F_{1, n-1}\left(u_{1}, \cdots, u_{r}\right) \mathcal{A}^{r-1}+F_{2, n-1}\left(u_{1}, \cdots, u_{r}\right) \mathcal{A}^{r-2}+\cdots+F_{r, n-1}\left(u_{1}, \cdots, u_{r}\right) \mathcal{I}\right) \frac{t^{n}}{n !} Z_{0},
$$

and taking into account the above positions, it follows

$$
Z(t)=\sum_{n=0}^{\infty}\left(F_{1, n-1}\left(u_{1}, \cdots, u_{r}\right) Z_{0}^{r-1}+F_{2, n-1}\left(u_{1}, \cdots, u_{r}\right) Z_{0}^{r-2}+\cdots+F_{r, n-1}\left(u_{1}, \cdots, u_{r}\right) Z_{0}\right) \frac{t^{n}}{n !} .
$$

Then, Equation (3.4) immediately follows by introducing the vector function $\mathcal{F}_{n-1}$ and the matrix $\left(Z_{0}^{r-1}, \cdots, Z_{0}^{\prime}, Z_{0}\right)$ defined above.

Remark 4 Note that the convergence of the vectorial series in any compact set $K$ of the space $\left(u_{1}, \cdots, u_{r}\right)$ is guaranteed, as the components of $\mathcal{F}_{n-1}$ are polynomials of weight not exceeding $n-1$, and consequently are bounded in $\mathrm{K}$.

\subsection{The Continuous Case, Via the Riesz-Fantappiè Formula}

By using the Riesz-Fantappiè it is possible to avoid series expansions. Indeed, we can prove the following result.

Theorem 6 The solution of the Cauchy problem (3.3) can be found in the form

$$
Z(t)=\mathrm{e}^{t \mathcal{A}} Z_{0}=\sum_{h=0}^{r-1}\left[\frac{1}{2 \pi i} \sum_{j=0}^{r-h-1}(-1)^{j} u_{j}(t) \oint_{\gamma} \frac{\mathrm{e}^{\lambda} \lambda^{r-h-j-1}}{P(\lambda, t)} \mathrm{d} \lambda\right] \cdot t^{h} Z_{0}^{h},
$$

where we denoted by $u_{j}(t)=t^{j} u_{j}, \quad(j=0,1, \cdots, r)$ the invariants of $t \mathcal{A}$ and by $P(\lambda, t)=\lambda^{r}-u_{1}(t) \lambda^{r-1}+\cdots+u_{r-1}(t) \lambda+u_{r}(t)$ its characteristic polynomial.

Proof It is a straightforward application of the Riesz-Fantappiè formula, taking into account the definition of $Z_{0}^{h},(h=0,1, \cdots, r-1)$.

\section{Worked Examples}

We show that the above results are easier with respect to the methods usually presented in literature ([1] [2]). Our technique is as follows: if the matrix $\mathcal{A}_{r \times r}$ has a low dimension $(r \leq 4)$, its invariants can be computed directly by hand. If $r \geq 5$ it is more easy to compute the eigenvalues by using one of the classical numerical methods, and then the invariants are found as the elementary symmetric functions (with alternate sign) of the eigenvalues. This completely avoids the construction of the Jordan canonical form. No necessity to compute higher powers of matrix $\mathcal{A}$. 


\subsection{Example 1 (Discrete Time Case)}

We consider the $3 \times 3$ system

$$
\left\{\begin{array}{l}
x_{n+1}=\frac{4}{5} x_{n}+\frac{3}{10} y_{n}+\frac{3}{10} z_{n} \\
y_{n+1}=\frac{1}{20} x_{n}+\frac{11}{20} y_{n}+\frac{1}{20} z_{n} \\
z_{n+1}=\frac{3}{20} x_{n}+\frac{3}{20} y_{n}+\frac{13}{20} z_{n}
\end{array}\right.
$$

with matrix $\mathcal{A}$ :

$$
\mathcal{A}:=\frac{1}{20}\left(\begin{array}{ccc}
16 & 6 & 6 \\
1 & 11 & 1 \\
3 & 3 & 13
\end{array}\right) .
$$

The invariants of $\mathcal{A}$ are by definition:

$$
\begin{gathered}
u_{1}:=\operatorname{tr}(\mathcal{A})=2, \\
u_{2}:=\frac{1}{2}\left[\operatorname{tr}(\mathcal{A})^{2}-\operatorname{tr}\left(\mathcal{A}^{2}\right)\right]=\frac{5}{4}, \\
u_{3}:=\operatorname{det}(\mathcal{A})=\frac{1}{4} .
\end{gathered}
$$

We will consider, the initial conditions:

$$
\left\{\begin{array}{l}
x_{0}=\frac{1}{5} \\
y_{0}=\frac{2}{5} \\
z_{0}=\frac{2}{5}
\end{array}\right.
$$

Then, as a consequence, we have:

$$
\left\{\begin{array}{l}
x_{1}=\frac{4}{5} x_{0}+\frac{3}{10} y_{0}+\frac{3}{10} z_{0}=\frac{2}{5}, \\
y_{1}=\frac{1}{20} x_{0}+\frac{11}{20} y_{0}+\frac{1}{20} z_{0}=\frac{1}{4}, \\
z_{1}=\frac{3}{20} x_{0}+\frac{3}{20} y_{0}+\frac{13}{20} z_{0}=\frac{7}{20},
\end{array}\right.
$$

and

$$
\left\{\begin{array}{l}
x_{2}=\frac{4}{5} x_{1}+\frac{3}{10} y_{1}+\frac{3}{10} z_{1}=\frac{1}{2}, \\
y_{2}=\frac{1}{20} x_{1}+\frac{11}{20} y_{1}+\frac{1}{20} z_{1}=\frac{7}{40}, \\
z_{2}=\frac{3}{20} x_{1}+\frac{3}{20} y_{1}+\frac{13}{20} z_{1}=\frac{13}{40}
\end{array} .\right.
$$

Starting from the initial conditions:

$$
\begin{array}{lll}
F_{1,-1}=0 & F_{1,0}=0 & F_{1,1}=1, \\
F_{2,-1}=0 & F_{2,0}=1 & F_{2,1}=0, \\
F_{3,-1}=1 & F_{3,0}=0 & F_{3,1}=0,
\end{array}
$$


and by means of the recurrence relation

$$
F_{k, n-1}\left(u_{1}, u_{2}, u_{3}\right)=2 F_{k, n-2}\left(u_{1}, u_{2}, u_{3}\right)-\frac{5}{4} F_{k, n-3}\left(u_{1}, u_{2}, u_{3}\right)+\frac{1}{4} F_{k, n-4}\left(u_{1}, u_{2}, u_{3}\right),
$$

with $k=1,2,3$ and $n \geq 0$, we find the following solution of the discrete dynamical system problem (4.1)-(4.2)

$$
\begin{aligned}
& x_{n}=F_{1, n-1} x_{2}+F_{2, n-1} x_{1}+F_{3, n-1} x_{0} \\
& y_{n}=F_{1, n-1} y_{2}+F_{2, n-1} y_{1}+F_{3, n-1} y_{0} \\
& z_{n}=F_{1, n-1} z_{2}+F_{2, n-1} z_{1}+F_{3, n-1} z_{0} .
\end{aligned}
$$

The (4.4) coincides with the following solution of the problem (4.1)-(4.2) obtained with the classical method of eigenvalues

$$
\begin{aligned}
& x_{n}=\frac{3}{5}-\frac{2}{5}\left(\frac{1}{2}\right)^{n}, \\
& y_{n}=\frac{1}{10}+\frac{3}{10}\left(\frac{1}{2}\right)^{n}, \\
& z_{n}=\frac{3}{10}+\frac{1}{10}\left(\frac{1}{2}\right)^{n} .
\end{aligned}
$$

\subsection{Example 2 (Continuous Time Case)}

We consider the $3 \times 3$ system

$$
\left\{\begin{array}{l}
x^{\prime}(t)=4 x(t)-y(t)-2 z(t) \\
y^{\prime}(t)=2 x(t)+y(t)-2 z(t) \\
z^{\prime}(t)=5 x(t)-3 z(t)
\end{array}\right.
$$

with matrix $\mathcal{A}$ :

$$
\mathcal{A}:=\left(\begin{array}{ccc}
4 & -1 & -2 \\
2 & 1 & -2 \\
5 & 0 & -3
\end{array}\right) .
$$

The invariants of $\mathcal{A}$ are by definition:

$$
\begin{gathered}
u_{1}:=\operatorname{tr}(\mathcal{A})=2, \\
u_{2}:=\frac{1}{2}\left[\operatorname{tr}(\mathcal{A})^{2}-\operatorname{tr}\left(\mathcal{A}^{2}\right)\right]=1, \\
u_{3}:=\operatorname{det}(\mathcal{A})=2 .
\end{gathered}
$$

We will consider, the Cauchy problem with initial conditions:

$$
\left\{\begin{array}{l}
x(0)=x_{0}=1 \\
y(0)=y_{0}=2 \\
z(0)=z_{0}=-1
\end{array}\right.
$$

Then, as a consequence, we have:

$$
\left\{\begin{array}{l}
x_{0}^{\prime}:=x^{\prime}(0)=4 x_{0}-y_{0}-2 z_{0}=4, \\
y_{0}^{\prime}:=y^{\prime}(0)=2 x_{0}+y_{0}-2 z_{0}=6, \\
z_{0}^{\prime}:=z^{\prime}(0)=5 x_{0}-3 z_{0}=8
\end{array}\right.
$$

and 


$$
\left\{\begin{array}{l}
x_{0}^{\prime \prime}:=x^{\prime \prime}(0)=4 x_{0}^{\prime}-y_{0}^{\prime}-2 z_{0}^{\prime}=-6, \\
y_{0}^{\prime \prime}:=y^{\prime \prime}(0)=2 x_{0}^{\prime}+y_{0}^{\prime}-2 z_{0}^{\prime}=-2, \\
z_{0}^{\prime \prime}:=z^{\prime \prime}(0)=5 x_{0}^{\prime}-3 z_{0}^{\prime}=-4 .
\end{array}\right.
$$

Starting from the initial conditions (4.3) and by means of the recurrence relation

$$
F_{k, n-1}\left(u_{1}, u_{2}, u_{3}\right)=2 F_{k, n-2}\left(u_{1}, u_{2}, u_{3}\right)-F_{k, n-3}\left(u_{1}, u_{2}, u_{3}\right)+2 F_{k, n-4}\left(u_{1}, u_{2}, u_{3}\right),
$$

with $k=1,2,3$ and $n \geq 0$, we find the following first values for the generalized Lucas polynomials $F_{k, n}\left(u_{1}, u_{2}, u_{3}\right)$ :

$$
\begin{array}{lcccc}
F_{1,2}=2 & F_{1,3}=3 & F_{1,4}=6, & F_{1,5}=13, & \ldots \\
F_{2,2}=-1 & F_{2,3}=0 & F_{2,4}=1, & F_{2,5}=0, & \ldots \\
F_{3,2}=2 & F_{3,3}=4 & F_{3,5}=6, & F_{3,5}=12, & \cdots
\end{array}
$$

Here we compute an approximation of the solution of the Cauchy problem obtained by a suitable truncation of order $N$ of the Taylor expansion

$$
\begin{aligned}
& x(t) \approx x_{N}(t)=\sum_{n=0}^{N}\left[F_{1, n-1} x_{0}^{\prime \prime}+F_{2, n-1} x_{0}^{\prime}+F_{3, n-1} x_{0}\right] \frac{t^{n}}{n !} \\
& y(t) \approx y_{N}(t)=\sum_{n=0}^{N}\left[F_{1, n-1} y_{0}^{\prime \prime}+F_{2, n-1} y_{0}^{\prime}+F_{3, n-1} y_{0}\right] \frac{t^{n}}{n !} \\
& z(t) \approx z_{N}(t)=\sum_{n=0}^{N}\left[F_{1, n-1} z_{0}^{\prime \prime}+F_{2, n-1} z_{0}^{\prime}+F_{3, n-1} z_{0}\right] \frac{t^{n}}{n !}
\end{aligned}
$$

The exact solution of the Cauchy problem (4.5)-(4.6) is

$$
\begin{gathered}
x(t)=-\mathrm{e}^{2 t}+2 \cos t+6 \sin t, \\
y(t)=2 \cos t+6 \sin t, \\
z(t)=-\mathrm{e}^{2 t}+10 \sin t,
\end{gathered}
$$

such that we can compute, by using a Mathematica program, the approximation error obtained, for some values of $N$, in a fixed points $t$ of the real axes. For example for $t=15$ and $N=100$ we obtain

$$
\begin{gathered}
x_{N}(t)=-1.0686474581522079795 \times 10^{13}, \\
y_{N}(t)=2.3823512152250586472, \\
z_{N}(t)=-1.0686474581517959268 \times 10^{13}, \\
\left|x(t)-x_{N}(t)\right|=2.3199126939274252816 \times 10^{-11}, \\
\left|y(t)-y_{N}(t)\right|=-3.6140640499302640175 \times 10^{-41}, \\
\left|z(t)-z_{N}(t)\right|=2.3199126939274252816 \times 10^{-11} .
\end{gathered}
$$

\subsection{Example 3 (Continuous Time Case)}

We consider the $4 \times 4$ system

$$
\left\{\begin{array}{l}
x_{1}^{\prime}(t)=x_{1}(t)-x_{2}(t)+3 x_{3}(t)-2 x_{4}(t) \\
x_{2}^{\prime}(t)=2 x_{1}(t)+x_{2}(t)-2 x_{3}(t)+x_{4}(t) \\
x_{3}^{\prime}(t)=-3 x_{1}(t)+4 x_{2}(t)+x_{3}(t)+2 x_{4}(t) \\
x_{4}^{\prime}(t)=-2 x_{1}(t)+5 x_{2}(t)-2 x_{4}(t)
\end{array}\right.
$$


with matrix $\mathcal{A}$ :

$$
\mathcal{A}:=\left(\begin{array}{cccc}
1 & -1 & 3 & -2 \\
2 & 1 & -2 & 1 \\
-3 & 4 & 1 & 2 \\
-2 & 5 & 0 & -2
\end{array}\right)
$$

The invariants of $\mathcal{A}$ are:

$$
\begin{gathered}
u_{1}=1, \\
u_{2}=7, \\
u_{3}=-74, \\
u_{4}=-180,
\end{gathered}
$$

We will consider, the Cauchy problem with initial conditions:

$$
\left\{\begin{array}{l}
x_{1}(0)=x_{1,0}=-1 \\
x_{2}(0)=x_{2,0}=2 \\
x_{3}(0)=x_{3,0}=1 \\
x_{4}(0)=x_{4,0}=3
\end{array}\right.
$$

Then, as a consequence, we have:

$$
\begin{gathered}
\left\{\begin{array}{l}
x_{1,0}^{\prime}=x_{1}^{\prime}(0)=x_{1,0}-x_{2,0}+3 x_{3,0}-2 x_{4,0}=-6 \\
x_{2,0}^{\prime}=x_{2}^{\prime}(0)=2 x_{1,0}+x_{2,0}-2 x_{3,0}+x_{4,0}=1 \\
x_{3,0}^{\prime}=x_{3}^{\prime}(0)=-3 x_{1,0}+4 x_{2,0}+x_{3,0}+2 x_{4,0}=18 \\
x_{4,0}^{\prime}=x_{4}^{\prime}(0)=-2 x_{1,0}+5 x_{2,0}-2 x_{4,0}=6
\end{array}\right. \\
\left\{\begin{array}{l}
x_{1,0}^{\prime \prime}=x_{1}^{\prime \prime}(0)=x_{1,0}^{\prime}-x_{2,0}^{\prime}+3 x_{3,0}^{\prime}-2 x_{4,0}^{\prime}=35 \\
x_{2,0}^{\prime \prime}=x_{2}^{\prime \prime}(0)=2 x_{1,0}^{\prime}+x_{2,0}^{\prime}-2 x_{3,0}^{\prime}+x_{4,0}^{\prime}=-41 \\
x_{3,0}^{\prime \prime}=x_{3}^{\prime \prime}(0)=-3 x_{1,0}^{\prime}+4 x_{2,0}^{\prime}+x_{3,0}^{\prime}+2 x_{4,0}^{\prime}=52 \\
x_{4,0}^{\prime \prime}=x_{4}^{\prime \prime}(0)=-2 x_{1,0}^{\prime}+5 x_{2,0}^{\prime}-2 x_{4,0}^{\prime}=5,
\end{array}\right.
\end{gathered}
$$

and

$$
\left\{\begin{array}{l}
x_{1,0}^{\prime \prime \prime}=x_{1}^{\prime \prime \prime}(0)=x_{1,0}^{\prime \prime}-x_{2,0}^{\prime \prime}+3 x_{3,0}^{\prime \prime}-2 x_{4,0}^{\prime \prime}=222 \\
x_{2,0}^{\prime \prime \prime}=x_{2}^{\prime \prime \prime}(0)=2 x_{1,0}^{\prime \prime}+x_{2,0}^{\prime \prime}-2 x_{3,0}^{\prime \prime}+x_{4,0}^{\prime \prime}=-70 \\
x_{3,0}^{\prime \prime \prime}=x_{3}^{\prime \prime \prime}(0)=-3 x_{1,0}^{\prime \prime}+4 x_{2,0}^{\prime \prime}+x_{3,0}^{\prime \prime}+2 x_{4,0}^{\prime \prime}=-207 \\
x_{4,0}^{\prime \prime \prime}=x_{4}^{\prime \prime \prime}(0)=-2 x_{1,0}^{\prime \prime}+5 x_{2,0}^{\prime \prime}-2 x_{4,0}^{\prime \prime}=-285 .
\end{array}\right.
$$

Starting from the initial conditions:

$$
\begin{array}{llll}
F_{1,-1}=0 & F_{1,0}=0 & F_{1,1}=0, & F_{1,2}=1, \\
F_{2,-1}=0 & F_{2,0}=0 & F_{2,1}=1, & F_{2,2}=0, \\
F_{3,-1}=0 & F_{3,0}=1 & F_{3,1}=0, & F_{3,2}=0, \\
F_{4,-1}=1 & F_{4,0}=0 & F_{4,1}=0, & F_{4,2}=0,
\end{array}
$$

and by means of the recurrence relation

$$
\begin{aligned}
F_{k, n-1}\left(u_{1}, u_{2}, u_{3}, u_{4}\right)= & F_{k, n-2}\left(u_{1}, u_{2}, u_{3}, u_{4}\right)-7 F_{k, n-3}\left(u_{1}, u_{2}, u_{3}, u_{4}\right) \\
& -74 F_{k, n-4}\left(u_{1}, u_{2}, u_{3}, u_{4}\right)+180 F_{k, n-5}\left(u_{1}, u_{2}, u_{3}, u_{4}\right),
\end{aligned}
$$

with $k=1,2,3,4$ and $n \geq 0$, we find the following first values for the generalized Lucas polynomials 
$F_{k, n}\left(u_{1}, u_{2}, u_{3}, u_{4}\right):$

$$
\begin{aligned}
& F_{1,3}=1, F_{1,4}=-6, F_{1,5}=-87, F_{1,6}=61, F_{1,7}=1294, \cdots \\
& F_{2,3}=-7, F_{2,4}=-81, F_{2,5}=148, F_{2,6}=1233, F_{2,7}=4931, \cdots \\
& F_{3,3}=-74, F_{3,4}=106, F_{3,5}=624, F_{3,6}=5358, F_{3,7}=-20174, \cdots \\
& F_{4,3}=180, F_{4,4}=180, F_{4,5}=-1080, F_{4,6}=-15660, F_{4,7}=10980, \cdots .
\end{aligned}
$$

Here we compute an approximation of the solution of the Cauchy problem obtained by a suitable truncation of the Taylor expansion

$$
\begin{aligned}
& x_{1}(t) \approx \sum_{n=0}^{8}\left[F_{1, n-1} x_{1,0}^{\prime \prime}+F_{2, n-1} x_{1,0}^{\prime}+F_{3, n-1} x_{1,0}\right] \frac{t^{n}}{n !} \\
&=-1-6 t+17.5 t^{2}+37 t^{3}+10.0417 t^{4}-41.525 t^{5} \\
&-23.3306 t^{6}+7.97798 t^{7}+14.1348 t^{8}, \\
& x_{2}(t) \approx \sum_{n=0}^{8}\left[F_{1, n-1} x_{2,0}^{\prime \prime}+F_{2, n-1} x_{2,0}^{\prime}+F_{3, n-1} x_{2,0}\right] \frac{t^{n}}{n !} \\
&=2+t-20.5 t^{2}-11.6667 t^{3}+20.9583 t^{4}+35.0583 t^{5} \\
&- 2.10278 t^{6}-16.0288 t^{7}-7.21639 t^{8}, \\
& x_{3}(t) \approx \sum_{n=0}^{8}\left[F_{1, n-1} x_{3,0}^{\prime \prime}+F_{2, n-1} x_{3,0}^{\prime}+F_{3, n-1} x_{3,0}\right] \frac{t^{n}}{n !} \\
&= 1+18 t+26 t^{2}-34.5 t^{3}-71.7917 t^{4}-7.35 t^{5} \\
&+49.8014 t^{6}+26.2446 t^{7}-9.01781 t^{8}, \\
& x_{4}(t) \approx \sum_{n=0}^{8}\left[F_{1, n-1} x_{4,0}^{\prime \prime}+F_{2, n-1} x_{4,0}^{\prime}+F_{3, n-1} x_{4,0}\right] \frac{t^{n}}{n !} \\
&= 3+6 t+2.5 t^{2}-47.5 t^{3}-9.33333 t^{4}+20.675 t^{5} \\
&+36.1653 t^{6}-5.16905 t^{7}-10.7202 t^{8} .
\end{aligned}
$$

\subsection{Example 4 (Using the Riesz-Fantappiè Formula)}

Consider the problem

$$
\left\{\begin{array}{l}
Z^{\prime}(t)=\mathcal{A} \cdot Z(t) \\
Z(0)=Z_{0}=(1,2)^{\mathrm{T}},
\end{array}\right.
$$

with matrix

$$
A=\left(\begin{array}{ll}
2 & 1 \\
1 & 2
\end{array}\right), \quad \text { then } \quad t \mathcal{A}=\left(\begin{array}{cc}
2 t & t \\
t & 2 t
\end{array}\right)
$$

Characteristic polynomial

$$
P(\lambda, t)=\lambda^{2}-4 \lambda t+3 t^{2}
$$

Matrix eigenvalues

$$
\lambda_{1}=t, \quad \lambda_{2}=3 t
$$

Matrix invariants

$$
u_{0}=1, \quad u_{1}=4 t, \quad u_{2}=3 t^{2} .
$$

From the initial condition 


$$
Z_{0}=Z_{0}^{0}=(1,2)^{\mathrm{T}}
$$

we find

$$
Z_{0}^{\prime}=\mathcal{A} Z_{0}=(4,5)^{\mathrm{T}} .
$$

Riesz-Fantappiè formula

$$
Z(t)=\mathrm{e}^{\mathrm{tA}} Z_{0}=\sum_{h=0}^{1}\left[\frac{1}{2 \pi i} \sum_{j=0}^{1-h}(-1)^{j} u_{j} \oint_{\gamma} \frac{\mathrm{e}^{\lambda} \lambda^{1-h-j}}{P(\lambda, t)} \mathrm{d} \lambda\right] \cdot t^{h} Z_{0}^{h} .
$$

i.e.

$$
\begin{aligned}
Z(t)=(x(t), y(t))^{\mathrm{T}}= & {\left[\frac{1}{2 \pi i} \sum_{j=0}^{1}(-1)^{j} u_{j} \oint_{\gamma} \frac{\mathrm{e}^{\lambda} \lambda^{1-j}}{\lambda^{2}-4 t \lambda+3 t^{2}} \mathrm{~d} \lambda\right] Z_{0}^{0} } \\
& +\left[\frac{1}{2 \pi i} \oint_{\gamma} \frac{\mathrm{e}^{\lambda}}{\lambda^{2}-4 t \lambda+3 t^{2}} \mathrm{~d} \lambda\right] \cdot t Z_{0}^{\prime}, \\
Z(t)=(x(t), y(t))^{\mathrm{T}}= & {\left[\frac{1}{2 \pi i} \oint_{\gamma} \frac{\mathrm{e}^{\lambda}(\lambda-4 t)}{\lambda^{2}-4 t \lambda+3 t^{2}} \mathrm{~d} \lambda\right] \cdot(1,2)^{\mathrm{T}} } \\
& +\left[\frac{1}{2 \pi i} \oint_{\gamma} \frac{\mathrm{e}^{\lambda}}{\lambda^{2}-4 t \lambda+3 t^{2}} \mathrm{~d} \lambda\right] \cdot(4,5)^{\mathrm{T}} .
\end{aligned}
$$

Integrals computation (using the Residue Theorem).

$$
\begin{aligned}
& {\left[\frac{1}{2 \pi i} \oint_{\gamma} \frac{\mathrm{e}^{\lambda}(\lambda-4 t)}{\lambda^{2}-4 t \lambda+3 t^{2}} \mathrm{~d} \lambda\right]} \\
& =\lim _{\lambda \rightarrow 3 t}(\lambda-3 t) \frac{\mathrm{e}^{\lambda}(\lambda-4 t)}{(\lambda-t)(\lambda-3 t)}+\lim _{\lambda \rightarrow t}(\lambda-t) \frac{\mathrm{e}^{\lambda}(\lambda-4 t)}{(\lambda-t)(\lambda-3 t)} \\
& =-\frac{\mathrm{e}^{3 t}}{2}+\frac{3 \mathrm{e}^{t}}{2}, \\
& {\left[\frac{1}{2 \pi i} \oint_{\gamma} \frac{\mathrm{e}^{\lambda}}{\lambda^{2}-4 t \lambda+3 t^{2}} \mathrm{~d} \lambda\right]} \\
& =\lim _{\lambda \rightarrow 3 t}(\lambda-3 t) \frac{\mathrm{e}^{\lambda}}{(\lambda-t)(\lambda-3 t)}+\lim _{\lambda \rightarrow t}(\lambda-t) \frac{\mathrm{e}^{\lambda}}{(\lambda-t)(\lambda-3 t)} \\
& =\frac{\mathrm{e}^{3 t}}{2 t}-\frac{\mathrm{e}^{t}}{2 t} .
\end{aligned}
$$

Solution of the problem

$$
Z(t)=(x(t), y(t))^{\mathrm{T}}=\left(-\frac{\mathrm{e}^{3 t}}{2}+\frac{3 \mathrm{e}^{t}}{2}\right) \cdot(1,2)^{\mathrm{T}}+\left(\frac{\mathrm{e}^{3 t}}{2 t}-\frac{\mathrm{e}^{t}}{2 t}\right) \cdot t \cdot(4,5)^{\mathrm{T}},
$$

i.e.

$$
\begin{aligned}
& x(t)=\left(-\frac{\mathrm{e}^{3 t}}{2}+\frac{3 \mathrm{e}^{t}}{2}\right)+4\left(\frac{\mathrm{e}^{3 t}}{2}-\frac{\mathrm{e}^{t}}{2}\right)=\frac{3 \mathrm{e}^{3 t}-\mathrm{e}^{t}}{2}, \\
& y(t)=2\left(-\frac{\mathrm{e}^{3 t}}{2}+\frac{3 \mathrm{e}^{t}}{2}\right)+5\left(\frac{\mathrm{e}^{3 t}}{2}-\frac{\mathrm{e}^{t}}{2}\right)=\frac{3 \mathrm{e}^{3 t}+\mathrm{e}^{t}}{2} .
\end{aligned}
$$

Checking our result 


$$
\begin{gathered}
x^{\prime}(t)=\frac{9 \mathrm{e}^{3 t}-\mathrm{e}^{t}}{2}, \quad y^{\prime}(t)=\frac{9 \mathrm{e}^{3 t}+\mathrm{e}^{t}}{2} . \\
x^{\prime}(t)=2 x(t)+y(t)=\frac{6 \mathrm{e}^{3 t}-2 \mathrm{e}^{t}}{2}+\frac{3 \mathrm{e}^{3 t}+\mathrm{e}^{t}}{2}=\frac{9 \mathrm{e}^{3 t}-\mathrm{e}^{t}}{2}, \\
y^{\prime}(t)=x(t)+2 y(t)=\frac{3 \mathrm{e}^{3 t}-\mathrm{e}^{t}}{2}+\frac{6 \mathrm{e}^{3 t}+2 \mathrm{e}^{t}}{2}=\frac{9 \mathrm{e}^{3 t}+\mathrm{e}^{t}}{2} .
\end{gathered}
$$

\section{Conclusions}

We have recalled that the exponential $\exp (\mathcal{A})$ of a matrix $\mathcal{A}$ can be written as a matrix polynomial, obtained from the scalar polynomial interpolating $\exp (x)$ on the spectrum of $\mathcal{A}$, and then avoiding the Taylor expansion for the exponential matrix.

By using the functions $F_{k, n}$, and in particular the fundamental solution of a homogeneous linear recurrence relation, i.e. the generalized Lucas polynomials of the second kind, we have shown how to obtain the solution of vectorial dynamical problems, both in the discrete (3.1) and continuous (3.3) case, in terms of functions of the invariants of $\mathcal{A}$, instead of powers of $\mathcal{A}$. These functions are independent of the Jordan canonical form of $\mathcal{A}$, and can be computed recursively, avoiding the knowledge of eigenvectors and principal vectors of the considered matrices. Moreover, if the matrix is real, the $F_{k, n}$ functions are real as well, and complex eigenvalues do not affect the form of the solution.

Furthermore, the use of the Riesz-Fantappiè formula (Sections 3.3 and 4.4) reduces to a finite computation the algorithms used in literature.

Therefore, the methods considered in this article are more convenient, with respect to those usually found in literature, for solving linear dynamical systems.

\section{References}

[1] Hirsch, M.W., Smale, S. and Devaney, R.L. (2003) Differential Equations, Dynamical Systems \& An Introduction to Chaos. Academic Press, (Elsevier), San Diego - London.

[2] Scheinerman, E.R. (2012) Invitation to Dynamical Systems. Dover, New York.

[3] Lucas, É. (1891) Théorie des Nombres. Gauthier-Villars, Paris.

[4] Raghavacharyulu, I.V.V. and Tekumalla, A.R. (1972) Solution of the Difference Equations of Generalized Lucas Polynomials. Journal of Mathematical Physics, 13, 321-324. http://dx.doi.org/10.1063/1.1665978

[5] Bruschi, M. and Ricci, P.E. (1982) An Explicit Formula for $f(\mathcal{A})$ and the Generating Function of the Generalized Lucas Polynomials. SIAM Journal on Mathematical Analysis, 13, 162-165. http://dx.doi.org/10.1137/0513012

[6] Bruschi, M. and Ricci, P.E. (1980) I polinomi di Lucas e di Tchebycheff in più variabili. Rendiconti di Matematica, 13, 507-530.

[7] Ricci, P.E. (1976) Sulle potenze di una matrice. Rendiconti di Matematica, 9, 179-194.

[8] Gantmacher, F.R. (1960) Matrix Theory. Chelsea Pub. Co., New York.

[9] Dunford, N. and Schwartz, J.T. (1988) Linear Operators, Part I General Theory. Wiley-Interscience, Hoboken. 